\title{
Design and proof of concept for multi degree of freedom Hydrostatically Coupled Dielectric Elastomer Actuators with roto- translational kinematics for object handling
}

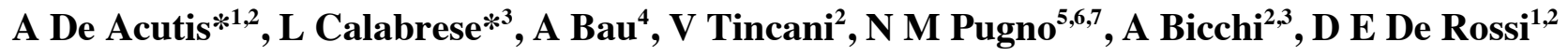 \\ ${ }^{1}$ Department of Information Engineering, University of Pisa, Via Girolamo Caruso 16, 56122 Pisa, Italy.
}

${ }^{2}$ SoftBots Lab, Istituto Italiano di Tecnologia, Via Morego 30, 16163, Genova, Italy.

${ }^{3}$ Department of Civil, Environmental and Mechanical Engineering, University of Trento, Via Mesiano 77, 38123 Trento, Italy.

${ }^{4}$ Research Centre "E. Piaggio”, University of Pisa, Largo Lucio Lazzarino 2, 56122 Pisa, Italy.

${ }^{5}$ Laboratory of Bio-Inspired and Graphene Nanomechanics, Department of Civil, Environmental and Mechanical

Engineering, University of Trento, 38123 Trento, Italy.

${ }^{6}$ School of Engineering and Materials Science, Queen Mary University of London, Mile End Road, E1 4NS London, United Kingdom.

${ }^{7}$ Ket Lab, Edoardo Amaldi Foundation, Italian Space Agency, Via del Politecnico snc, 00133 Rome, Italy.

* Both authors contributed equally to this manuscript

E-mail: aurora.deacutis@for.unipi.it

\begin{abstract}
In this article we present an upgraded design of the existing push-pull Hydrostatically Coupled Dielectric Elastomer Actuator (HC-DEA) for use in the field of soft manipulators. The new design has segmented electrodes, which stand as four independent elements on the active membrane of the actuator. When properly operated, the actuator can generate both out of plane and in plane motions resulting in a multi-degrees of freedom soft actuator able to exert both normal pushes (like a traditional HC-DEA) and tangential thrusts. This novel design makes the actuator suitable for delicate flat object transportation. In order to use the actuator in soft systems, we experimentally characterized its electromechanical transduction and modeled its contact mechanics. Finally, we show that the proposed actuator can be employed as a modular unit to develop active surfaces for flat object roto-translation.
\end{abstract}

Keywords: Soft platform, soft robotics, dielectric elastomer actuator, object transportation. 


\section{Smart Materials \& Structures}

\section{Introduction}

Material handling (e.g. grasping, transportation and sorting) is very important in all productive activities, particularly in industrial fields and in robotic industry worldwide. Usually, in manufacturing facilities, part feeders and conveyor systems together with grippers and manipulators are used for picking, sorting and moving items between specific locations [1]. All these systems are required to be effective, robust, fast and reliable in order to guarantee a rapid order fulfillment; yet, size and structural rigidity often limit their use, especially when the handling of fragile and/or delicate objects is required [2] or when frequent changes of picking and delivery locations occur.

To address the issue of fragile and delicate object handling, the growing research field of soft robotics has provided many devices that are based upon different concepts with respect to the more traditional rigid robotics. They rely on soft and compliant actuation systems such as pneumatic drives and variable stiffness actuators, on intrinsically adaptive structures that feature under-actuated mechanism and on the employment of soft materials that range from simply deformable to smart engineered materials (such as shape memory alloy and electro active polymers) [3], [4]. By exploiting these principles, many soft grippers and manipulators capable of stably and gently handling unknown shaped objects have been designed and reported in literature. One of the most representative soft gripper is the Universal Gripper, which stands out for its simplicity and adaptability to the shape of the grasped objects. It is composed of granular material encased in a soft and elastic membrane that allows for deforming and adapting to the shape of the objects as it is pushed against them. Making a positive or negative pressure inside the membrane the gripper can quickly grip and release a broad range of objects of different shapes. The adaptability of the Universal Gripper allows for distributing the grasping force over a larger surface of contact than a not adaptive soft gripper does. Furthermore, by tuning the intensity of the pressure, it allows to modulate the grasping force making it particularly suitable also for delicate parts [5]. All these features contributed to make it a very successful commercial product of the last years demonstrating how much desirable those characteristics are for industry. Although in the last decade many soft grippers have been designed, on the other hand, the use of soft-bodied conveyors or feeders for the transportation of delicate objects rather than the traditional rigid-bodied ones is still a rather unexplored field.

Indeed, the soft-bodied conveyors or feeders reported in literature are mostly shaped as pneumatically deformable soft surfaces capable of moving objects on a horizontal plane. In this regard, key examples are the fluid-based systems such as the "Festo Wavehandling" platform [6], which features a soft surface actuated by a pneumatic system that enables for the displacement of round objects through a wave-like motion, and the soft platform developed by Stommel et al. [7], featuring a pneumatically-induced peristaltic motion enabling it for ovine offal sorting. Another interesting device for material handling is described in the work of Mosadegh 


\section{Smart Materials \& Structures}

et al. [8] who presented a reconfigurable manifold designed for controlling pneumatically actuated soft machines, based on a computer-controlled braille display. Such device relies on a compact array of piezoelectric actuators, and on an interchangeable micro pneumatic system, allowing it for controlling a large number of independent outputs. Since these systems are properly designed for specific purposes their usage is limited only to round objects with approximately spherical or, at most, cylindrical shape. To address this limitation, Deng et al. [9] proposed a pneumatic machine table for manipulation of delicate objects inspired by caterpillar locomotion. This machine table successfully tackled the challenge of displacing flat objects like an iPhone in both translational and rotational direction on a plane, thanks to a peculiar shape and actuation control logic of its inflatable chambers, driven by an external pumping system. Despite these relevant attempts to improve the performance and usability of these systems, pneumatic-based machines still require a complex design, a cumbersome and expensive external pneumatic hardware (e.g. valves, pumps and compressors) and they also feature a high energy consumption, thus making them unsuitable in many applications [10].

In order to exploit a more convenient actuation principle, Wang et al. made use of the Dielectric Elastomer (DE) actuation technology to develop a soft-wave handling system [2], featuring a matrix of 16 air-filled bubble-like actuators similar to the fluid-filled push-pull Hydrostatically Coupled Dielectric Elastomer Actuators (HC-DEAs) first presented by Carpi et al. [11]. It is worth to point out that such bubble-like actuators are built by coupling two pre-stretched membranes enclosing a finite volume of air or fluid sealed upon manufacturing; therefore they do not require any pumping system for their actuation. However, since such devices work by design only in push and pull modes [11], when embedded in a soft platform, they are only capable to trigger the rolling of round objects placed on the platform's surface (as shown in [2]) but are unsuitable to induce the in-plane displacement of any object that can not roll, such as a flat object, since they are not capable of generating any tangent thrust but only a normal one.

Aimed at overcoming this limitation, we designed a new concept of HC-DEA with independent segmented electrodes on the active membrane (as better described in the following Sec. 2), which enables the actuator to generate both normal and tangent thrusts on objects placed in contact with its surface. Although the use of segmented electrodes in dielectric elastomer actuators was already reported to be effective for the conical DEbased multi-DoF actuator, presented by Conn and Rossiter [12], to the best of the author's knowledge, it has never been used for HC-DEA systems. Indeed, this simple change in the design of the electrodes results in a significant enhancement of the HC-DEA capabilities, making it able to generate both an out of plane action (push-pull behavior) and an in-plane one. In this way the actuator can operate following infinite trajectories in the space, therefore we called it multi-Degree of Freedom (multi-DoF) HC-DEA. Thanks to the ability to exert both normal and tangential thrusts in any direction in a plane, the multi-DoF HC-DEA enables a variety of possible applications in the field of soft manipulation. In particular, with this work we intend to demonstrate 


\section{Smart Materials \& Structures}

the possibility of using it as a building block of a modular soft platform aimed at handling objects. Moreover, since each actuator of the platform can be independently controlled by an electric signal, the result is an easily reprogrammable and reconfigurable soft surface composed by a plane matrix of actuators allowing for both round and flat objects handling. The proposed multi-DoF HC-DEA was investigated in terms of electromechanical transduction performance and contact mechanics. Actuation experiments were also carried out, thus proving the capacity of an array of multi-DoF HC-DEAs to roto-translate flat objects on a horizontal surface.

This paper is organized as follows. Sec. 2 introduces the conceptual design and working principle of the multiDoF HC-DEA. Sec. 3 reports on the mechanical interaction between the multi-DoF HC-DEA and objects. Sec. 4 presents the roto-translation experiments performed with a flat object. Finally, Sec. 5 presents our conclusions.

\section{Conceptual design and working principle of the multi-DoF HC-DEA}

\subsection{Key idea}

Within the field of DE transducers, HC-DEAs are of particular interest nowadays. As described in [11], a conventional HC-DEA consists of two pre-stretched membranes coupled together by a fixed volume of an incompressible fluid (Fig. 1).

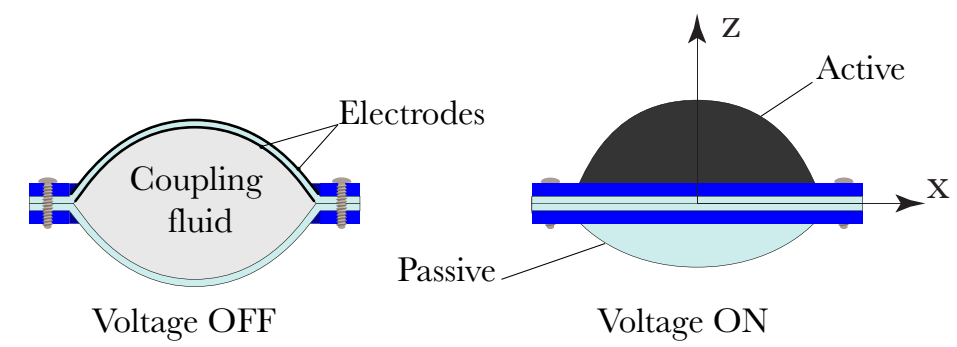

Fig. 1. A push-pull HC-DEA. In rest state (left) and in active state (right).

The upper membrane of the actuator is coated on the inner and the outer sides with compliant electrodes, which form a flexible DE capacitor. Due to the pre-stretch of the membranes, the coupling fluid is sealed between the membranes with an initial pressure. Such pressure keeps the membranes stretched, enhancing the dielectric breakdown strength [13] and thus the electromechanical transduction capability of the actuator. When a voltage difference is applied to the electrodes, an electrostatic pressure is generated between them, thereby reducing the thickness and expanding the area of the elastomeric material due to its incompressibility [14-16]. Accordingly, the active membrane expands outward, while the passive membrane is dragged inward, driven by the hydrostatic transmission of the intermediate fluid. Therefore, a conventional HC-DEA results in 


\section{Smart Materials \& Structures}

a push-pull device whose stroke depends on the applied voltage. Considering the active membrane's apical point as a reference, due to axis-symmetry upon voltage application, the point is displaced along the out-ofplane (z-axis) direction only, and therefore no in-plane displacement is allowed. We can thus refer to it as a one-degree of freedom actuator, which exerts only a normal force on an object in contact with its surface.

In order to enhance the functionality of the actuator with an in-plane displacement capability, we present an upgrade that features a particular electrode pattern (Fig. 2). The design of the new electrodes, which stand as four independent quarter-circles within the active membrane, enables a tridimensional trajectory of the apical point to be generated, as the driving voltage $V$ is applied. This new feature distinguishes the proposed actuator from the state of the art by allowing its active membrane for generating both a normal and a tangent thrust on the object to be moved. Indeed, when all the electrodes are activated the actuator behaves like a traditional push-pull HC-DEA, showing an axisymmetric out of plane deformation. On the other hand, when a voltage $V$ is applied to two adjacent electrodes, for instance on the right side as depicted in Fig. 2, only that part of the active membrane expands as a result of the applied voltage. Thus, this deformed configuration is not axisymmetric and the apical point moves to the left in addition to an out of plane displacement. In this fashion, the actuator is able to exert both a normal and a tangential (leftward in this example) thrust.

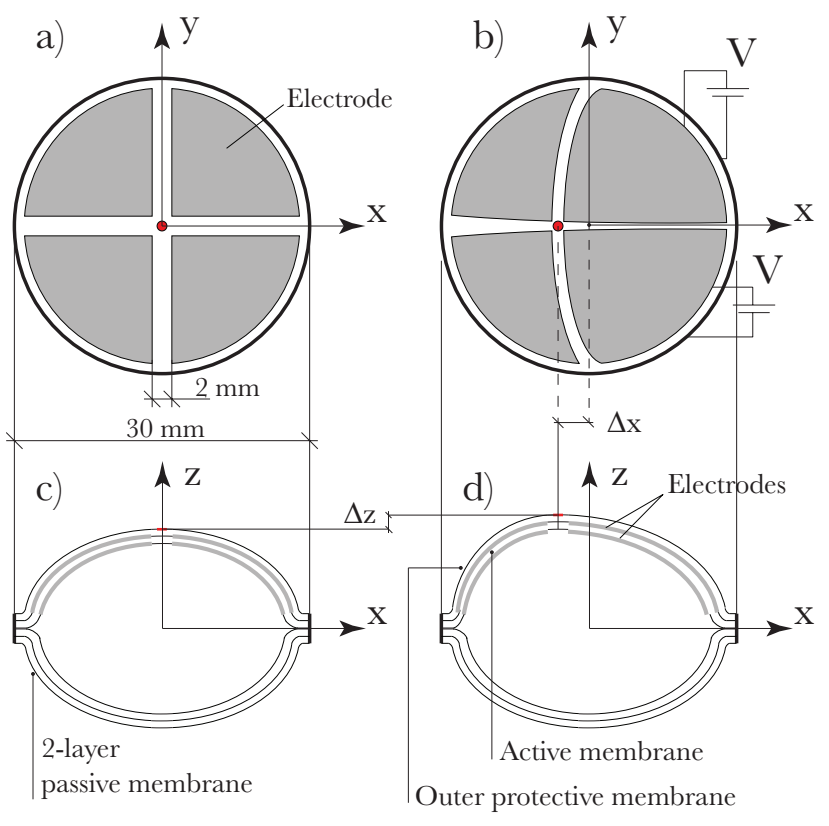

Fig. 2. Top view of the multi-DoF HC-DEA in its rest state (a) where the four independent electrodes enable the tridimensional displacement of the actuator's apical point. For example, when a driving voltage $V$ is applied to the two electrodes on the righthand side, a displacement is triggered along the $\mathrm{x}$-axis coupled to a displacement along the $\mathrm{z}$-axis (b). The electrode position within the coupled layers that constitute the active membrane of the actuator is also highlighted in the $\mathrm{X}$-Z section planes (c) and $(\mathbf{d})$. 


\section{Smart Materials \& Structures}

\subsection{Manufacturing}

The actuator was fabricated with an acrylic elastomer film (VHB 4910, 3M, USA) for both the active and the passive membranes, carbon conductive grease (Carbon Conductive Grease 846, M.G. Chemicals, Canada) for the electrodes and silicone grease (8462, M.G. Chemicals, Canada) for the coupling fluid. In order to isolate the electrodes from the external environment, thereby preventing possible damage occurring from the contact with objects, the active membrane was coupled with a second outer insulating VHB film, as shown in Fig. 2. In order to obtain an actuator with a symmetrical shape at rest, the same double layer configuration was used for the manufacturing of the passive membrane. The fabrication process of the actuator is described in Fig. 3. In particular, Fig. 3a describes the fabrication procedure of both the active and the passive membranes, in which two layers of VHB 4910 elastomer, each with an initial thickness of $1 \mathrm{~mm}$, were equi-biaxially prestretched by $300 \%$ and secured to individual support frames. Due to the incompressibility of the material, the surface expansion of each layer, induced upon prestretch, resulted in a thickness reduction from the original thickness of $1 \mathrm{~mm}$ to $62.5 \mu \mathrm{m}$. Carbon grease electrodes were smeared with a brush and a stencil on both sides of the first layer of the active membrane. Thanks to the high adhesive properties of the VHB elastomer, this first layer was coupled to the second VHB layer forming a perfect bonding on the surfaces not covered by the electrodes. Copper tape was used for connecting the electrodes to the power source. The same procedure was repeated for the fabrication of the double layer passive membrane, except for the phase of the electrode deposition.

The active and passive membranes were then coupled together by means of two 3D printed plastic frames (see Fig. 3b), and filled with a volume of silicone grease (8462, M.G. Chemicals, Canada). The grease was inserted using a syringe through a silicone hose placed between the membranes and secured to the plastic frames. Each actuator resulted in a bubble-like shaped structure filled with $7 \mathrm{ml}$ of coupling fluid, thus presenting a $30 \mathrm{~mm}$ diameter measured in relation to the horizontal plane of symmetry and a height of the apical point of $10 \mathrm{~mm}$ from the same plane. The driving voltage $V$ was provided to each electrode pair by thin copper strips with negligible thickness and stiffness, placed in direct contact with the electrodes.

In order for the multi-DoF HC-DEA to perform object displacement, the actuator was enclosed in the support structure represented in Fig. 3b. The structure provides a flat top surface to support the objects to be displaced, and features a $20 \mathrm{~mm}$ diameter hole through which the actuator reaches the contact with the object upon activation performing the actuation cycle described in the following. 


\section{Smart Materials \& Structures}

a) Mebranes manufacturing

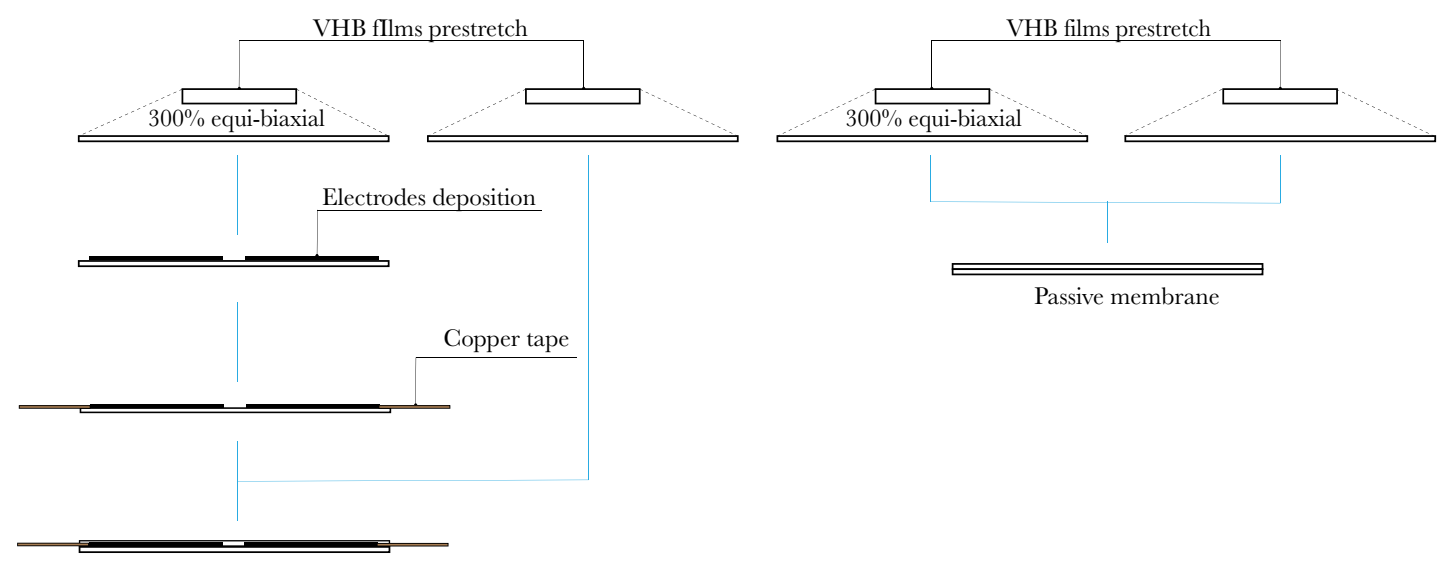

Active membrane

b) Multi-DoF HC-DEA manufacturing
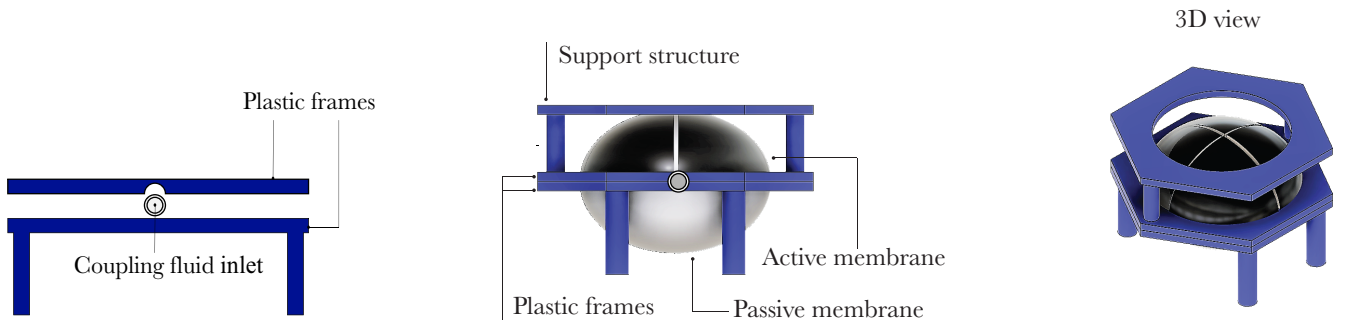

Fig.3. Schematic representation of the manufacturing process of the actuator. Fabrication procedure of both the active and the passive membranes (a), assembly of the actuator's components (b).

\subsection{Actuation cycle}

The actuation cycle adopted to enable the object to slide on the support surface is described in Fig. 4. The cycle is composed of four steps, namely A, B, C and D, specifically designed to trigger the object to slide along one direction of the plane (with reference to Fig.2 in this case along the x-axis from left to right).

At the beginning of the cycle, see Fig. 4a, the active membrane is in its rest state: no voltage is applied to the four electrodes of the actuator so that, when it is enclosed in the support structure, its apical point is just below the top surface of the support structure in incipient contact with the object.

In the second step, described in Fig. $4 \mathrm{~b}$, the actuator makes contact with the object thanks to the application of a driving voltage $V=3.5 \mathrm{kV}$ to the two electrodes on the right side, which generates both a vertical and a horizontal displacement of the apical point.

Figure $4 \mathrm{c}$ shows the third step of the cycle, where the driving voltage is applied to all four electrodes. During the transition between step B and C, the object can be lifted and displaced rightward from its original position. 


\section{Smart Materials \& Structures}

From this condition, in the last step of the cycle, see Fig. $4 d$, the voltage on the right side electrodes is switched off, inducing a further horizontal shift of the object and a drop in the vertical height to the final position. In this fashion, by repeating the actuation cycle a proper number of times, it is possible to achieve any desired horizontal displacement as sum of the displacement generated by one cycle.

It is worth to point out that, the same actuation cycle can be used to trigger the sliding of the object along the y-axis, simply by activating the two couples of electrodes along that direction. Finally, each electrode in the multi-DoF HC-DEA can be activated individually, by different voltage signals, thus enabling the actuator to follow infinite trajectories.

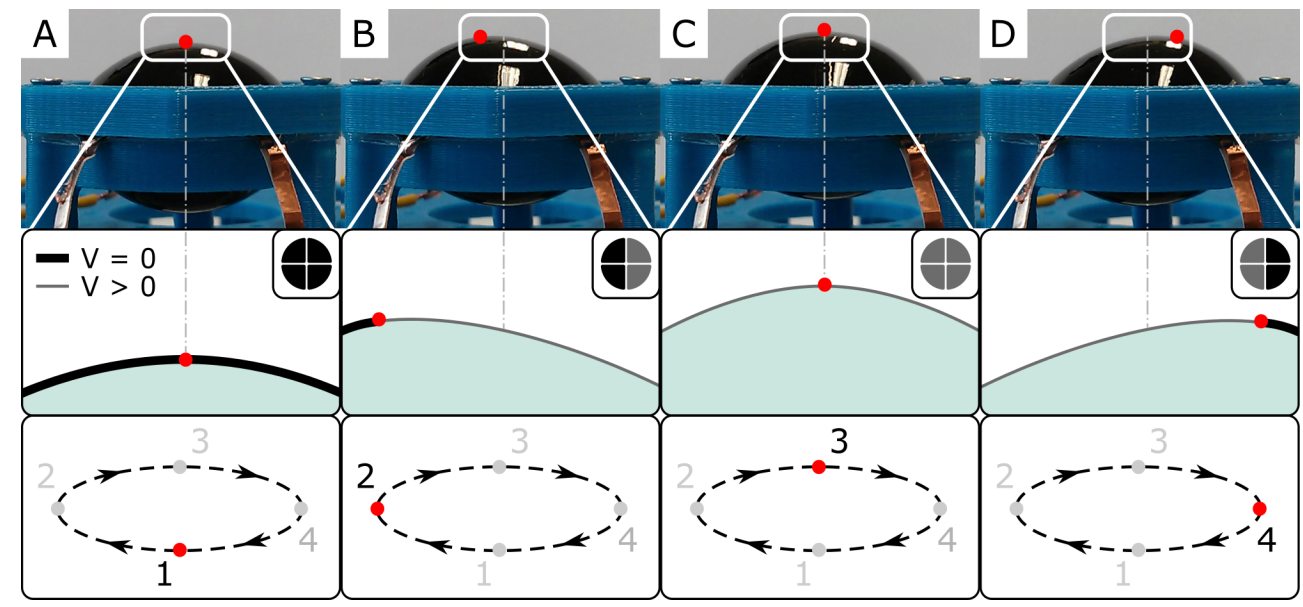

Fig. 4. Actuation cycle used to induce the unidirectional motion of a flat object. The first row shows the pictures of the multiDoF HC-DEA in each of the four steps of the actuation cycle (namely A, B, C and D), with its apical point identified by a red marker. A magnification of the voltage-induced deformation is shown in the second row, where the active membrane is represented by both the grey line $V>0$ and by the grey electrodes drawn in the logo (top right corner). The passive part is represented by the black bold line $V=0$ and by the black electrodes. The last row highlights the position of the apical point for each step in the cycle.

\section{Mechanical interaction between the multi-DoF HC-DEA and objects}

During the actuation cycle, the actuator reaches the contact with the object and moves it on the horizontal plane thanks to the friction force generated through the contact area. If no slide occurs, according to the Coulomb friction model, the magnitude of the friction force is a function of both the normal force and the static friction coefficient. Therefore, in order to calculate the friction force generated on the object at a given voltage $V$ it is important to know the normal force as function of the driving voltage $V$ and to identify the value of the static friction coefficient for the object to be moved. For this reason, we experimentally measured the static friction coefficient between the active membrane of the actuator and four surfaces of different materials. Also, a test was carried out to identify the relation between the applied voltage and the normal blocking force 


\section{Smart Materials \& Structures}

exerted by the actuator. Eventually, the actuator was characterized as soft manipulator in terms of the relation between a geometrical parameter of the contact area and the normal blocking force and compared with robotic soft fingers.

\subsection{Measurement of the static friction coefficient}

This subsection reports the measurements performed to calculate the static friction coefficient occurring between the surface of the actuator and four flat objects made of plastic, aluminum, plywood and rigid cardboard. In performing the measurements, the active membrane of the actuator was dusted with talcum powder in order to prevent pernicious adhesive effects (as required in the usual operational condition). A conceptual scheme of the experimental set-up carried out to measure the static friction coefficient between the multi-DoF HC-DEA and the material samples tested is described in Fig. 5. The test procedure is as follows. After securing the multi-DoF HC-DEA on a motorized linear carrier, which allowed for a translation along a horizontal axis, the first object was glued to a tri-axial ATI Nano 17 force sensor located on a second linear stage operating along a vertical axis over the actuator. Thanks to the linear stage, the sensor and the object were lowered until the object made contact with the actuator's active membrane. After setting an initial normal force of $0.1 \mathrm{~N}$, the horizontal linear stage was activated with a velocity of $0.5 \mathrm{~mm} / \mathrm{s}$. Using the sensor to record both the normal force $N$ and the tangential force $F_{f}$ at the time of incipient sliding, the static friction coefficient $\mu=F_{f} / N$ was calculated for each specimen tested, and reported in Tab. 1.

Table 1. Values of the static friction coefficient measured for different materials.

\begin{tabular}{cc}
\hline Material & Static friction coefficient $\mu$ \\
\hline ABS plastic & 0.63 \\
Aluminum & 0.72 \\
Cardboard & 0.76 \\
Plywood & 0.81 \\
\hline
\end{tabular}

These high values of the static friction coefficient $\mu$ allow the actuator to apply relatively high tangent forces on the object to be moved. Therefore, the actuator can operate with a gentle touch that makes it suitable for the displacement of delicate objects as better described in the following. 


\section{Smart Materials \& Structures}

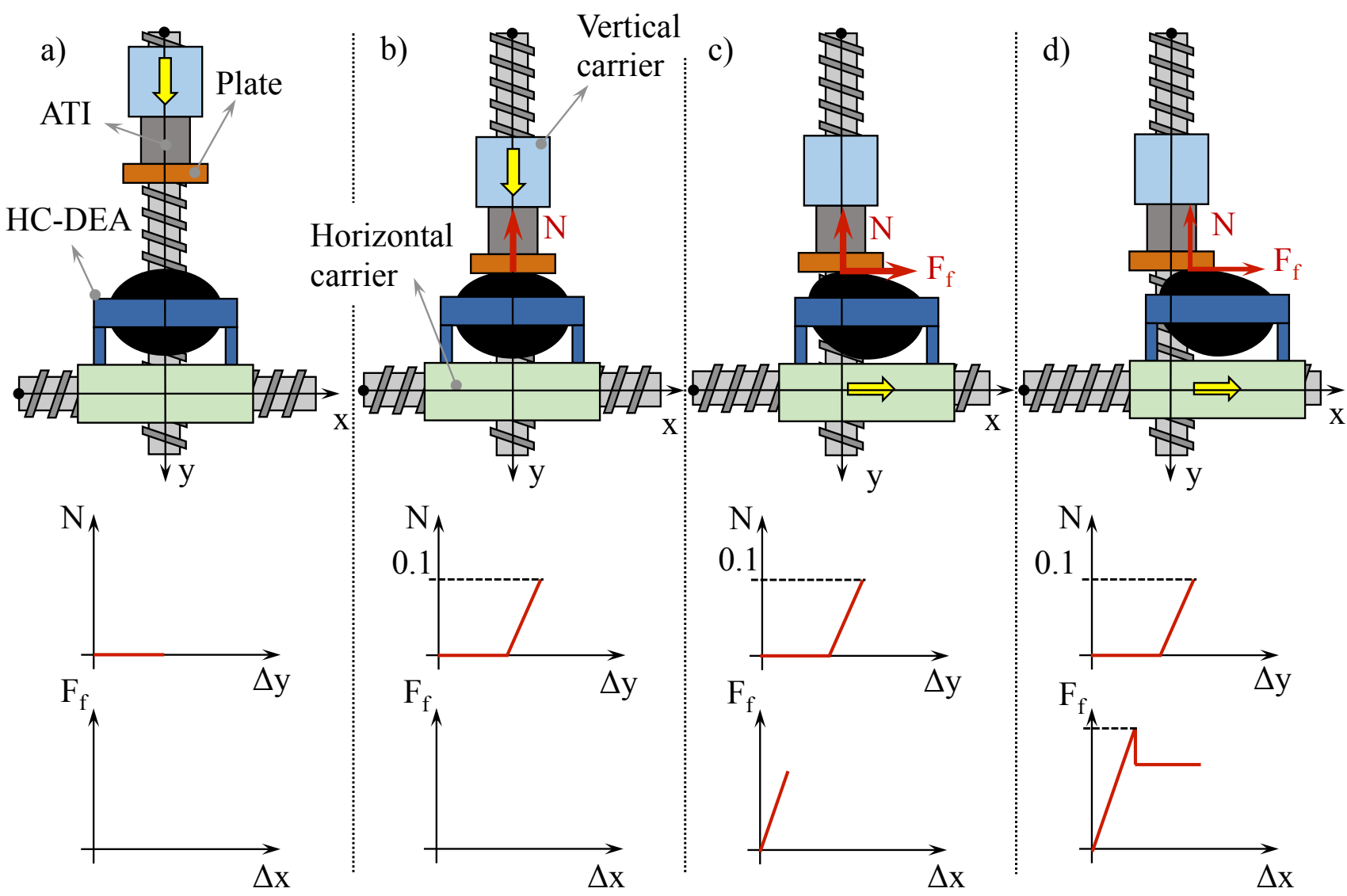

Fig. 5. Conceptual scheme of the experimental set-up carried out to measure the static friction coefficient between the HC-DEA and diverse materials. In the picture, the execution of the experiment is broken down into four phases. Each phase is furnished with a graph of the normal and tangential force as functions of the displacement $\Delta x$ and $\Delta y$ of the carriers with respect to their location at the first phase: (a) the vertical carrier, on which a 6-axis ATI-Nano force/torque sensor and a plate of an established material are mounted, start moving down along the y-axis; (b) the plate contacts the HC-DEA and the descent of the carrier stops when a normal force $N$ of $0.1 \mathrm{~N}$ is reached; (c) after $N$ reaches $0.1 \mathrm{~N}$, the horizontal carrier moves along the $\mathrm{x}$-axis at a constant velocity of $0.5 \mathrm{~mm} / \mathrm{s}$. A tangential force $F_{f}$ arises on the system composed of the plate and the ATI sensor through the contact area at the apical point of the HC-DEA which, in this stage, does not move with respect to the plate; (d) as the horizontal carrier moves rightwards, the tangential force increases until it reaches a peak value when the contact area is on the verge of moving. After the peak value, that is the maximum static friction force we seek, the contact area translates rightwards and $F_{f}$ slightly decreases at the value of the kinetic friction force.

\subsection{Electro-mechanical performance}

During phases B, C and D in the actuation cycle (Fig. 4), the active surface of the multi-DoF HC-DEA makes contact with the object and generates both normal and tangent forces. By modelling the pre-slide regime with the Coulomb friction model we obtain:

$$
F_{f} \leq \mu N,
$$




\section{Smart Materials \& Structures}

where $F_{f}$ is the friction force at the interface object-actuator, $\mu$ is the static friction coefficient and $N$ is the normal force at the interface object-actuator. Since the deformation of the multi-DoF HC-DEA is voltage dependent, the resulting normal and tangent blocking forces are functions of the voltage amplitude $V$. In order to identify such relations, an actuation test was performed with a double column dynamometer (Z005, Zwick Roell, Germany) featuring a measure precision of 0.001 N. Figure 6 shows the experimental setup.
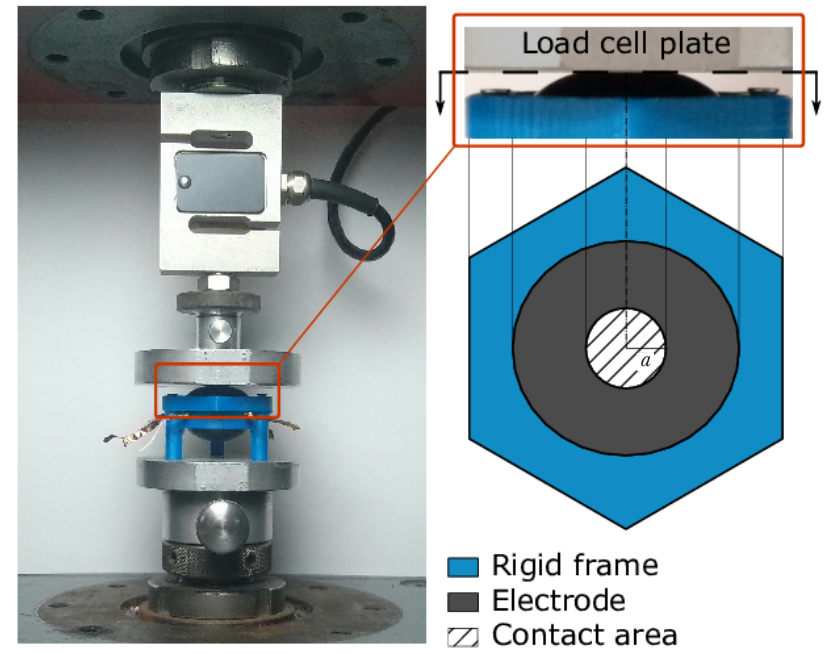

Fig. 6. Compression tests of the actuation unit: experimental set-up. The compression tests of the actuator were performed with a uniaxial testing machine (left) equipped with a load cell for force measurements. The contact area was approximated with a circle of radius $a$ (right).

The test procedure was as follows: 1) the multi-DoF HC-DEA actuator was placed on the horizontal surface of the uniaxial testing machine; 2) the load cell plate was lowered until an incipient contact with the actuator occurred; 3 ) the driving voltage $V$ was applied to the two electrodes on the right-hand side (so as to simulate steps B or D of the actuation cycle); 4) for each step of the voltage ramp, the readings of the normal force were recorded after $10 \mathrm{~s}$ from the voltage application. Fig. 7 shows the experimental data as dots on a $V$ - $N$ graph.

From Fig. 7 it turns out that the curve can be treated with a good approximation as a power function:

$$
N=f(V)=k V^{\beta}
$$

from which, through the knowledge of the coefficient of friction, we obtain the corresponding values of the tangent blocking force $F_{f}$ as:

$$
F_{f}=\mu N=\mu k V^{\beta}
$$

Performing a best fitting of the experimental data with the power function (2), the values $k=7.64610^{-10}$ $\mathrm{NV}^{-\beta}$ with $\beta=2.354$ were obtained, for $N$ expressed in $\mathrm{N}$ and $V$ in $\mathrm{V}$ [SI]. Fig. 7 shows the experimental data and the corresponding best fitting curve of both the normal blocking force $N$ and the tangent blocking 


\section{Smart Materials \& Structures}

force $F_{f}$ calculated for $\mu=0.63$. The graph shows that the maximum tangential thrust that a multi-DoF HCDEA can exert on a plastic object is around $0.1 \mathrm{~N}$ with a voltage of $3.5 \mathrm{kV}$.

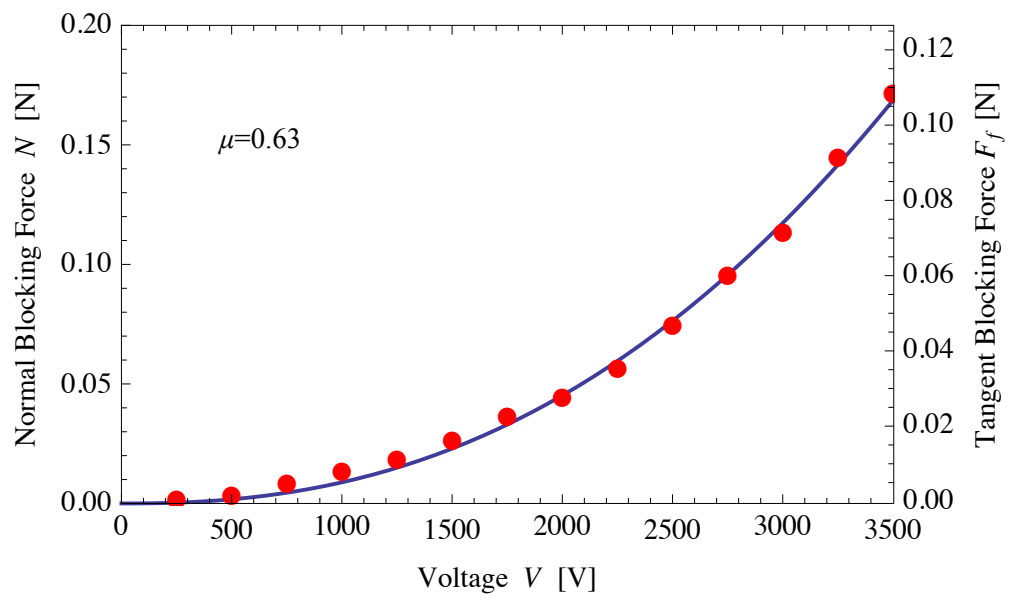

Fig. 7. The normal blocking force $N$ as a function of the driving voltage $V$, experimental data and best fitting function $N=f(V)=$ $7.64610^{-10} V^{2.354}$. The correspondent tangential blocking force $F_{f}=\mu f(V)$ is also reported for a static friction coefficient $\mu=0.63$ obtained for the contact with ABS plastic.

\subsection{Compliance of the active membrane}

When the active membrane of the actuator reaches the contact with an object the contact area increases with a non-linear function of the normal force $N$, and therefore of the driving voltage $V$. Since these relations provide information about the compliance of the actuator (e.g. allowing to calculate the contact pressure exerted at the interface), the contact area $A$ was measured as a function of the driving voltage $V$ by adding a further step to the test procedure reported in Sect. 3.2. Indeed, in this case the active membrane was smeared with a liquid colorant and a piece of graph paper was placed between the load cell plate and the actuator, allowing for measuring also the contact area imprints. For each step of voltage, the contact imprints were approximated by a best fitting circle calculated from a digital image analysis performed with MATLAB, with a $0.5 \mathrm{~mm}$ standard error on the estimate of the circle radius. The experimental data are plotted as dots in Fig.8. In order to compare the compliance of the HC-DEA with respect to other soft parts that are used as reference in soft robotics, it is worth approximating the nonlinear relation between the contact circle radius $a$ and the voltage $V$ with the best fitting power function:

$$
a=h(V)=k^{\prime} V^{\alpha},
$$

where $k^{\prime}=0.315 \mathrm{mmV}^{-\alpha}, \alpha=0.352 \mathrm{~mm}$ and $V$ is expressed in $\mathrm{V}$ [SI]. In Fig.8 the best fitting power function is drawn with a continuous line. 


\section{Smart Materials \& Structures}

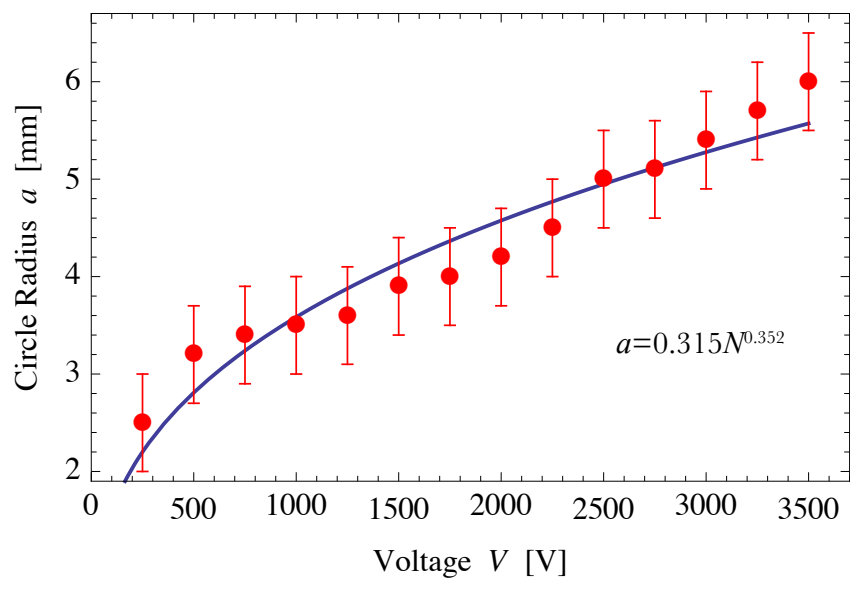

Fig. 8. The contact circle radius $a$ as a function of the driving voltage $V$, experimental data and best fitting function $a=h(V)=$ $0.315 V^{0.352}$.

Considering both the nonlinear relations $f(V)$ described in (2) and $h(V)$ reported in (4), it is possible to calculate the expression $a=g(N)=h\left(f^{-1}(N)\right)$ to obtain the nonlinear relation of the circle radius $a$ as a function of the normal force $N$ :

$$
a=g(N)=k^{\prime}\left(\frac{N}{k}\right)^{\alpha / \beta}=c N^{\gamma} .
$$

Where $c=\frac{k^{\prime}}{k^{\gamma}}$ and $\gamma=\alpha / \beta$. By using the coefficients $\alpha, \beta, k$ and $k^{\prime}$ previously defined we calculated $c=7.270 \mathrm{mmN}^{-\gamma}$ with $\gamma=0.149$, for $a$ expressed in $\mathrm{mm}$ and $N$ in $\mathrm{N}$ [SI], and consequently we obtained the nonlinear function $g(N)$ that relates the radius of the contact circle to the normal force $N$.

Indeed, this non-linearity between the normal force and contact area is common in contact mechanics, particularly in the case of soft silicone fingers performing grasping actions on rigid objects [17]. In order to assess the compliance of the multi-DoF HC-DEA to these soft silicone fingers a comparison is shown in Fig. 9. In particular Fig. 9 shows that the experimental data and the nonlinear power function $a=g(N)$ calculated for the multi-DoF HC-DEA are comprised between the two curves reported in [17], obtained for a soft silicone finger and a highly viscoelastic finger made of an artificial skin filled with gel. Figure 9 also shows that in the contact between the multi-DoF HC-DEA and a flat object, for small values of the normal force $N$ the rate of increase of the contact area slows down as the normal force increases, whereas for higher values the rate of increase tend to be constant. This behavior is also observed in soft materials, such as rubber and silicone, as well as in human fingers $[17,18]$. Thus, the multi-DoF HC-DEA makes a large area of contact for small normal forces, ensuring a gentle touch, which makes it suitable for handling delicate objects. 


\section{Smart Materials \& Structures}

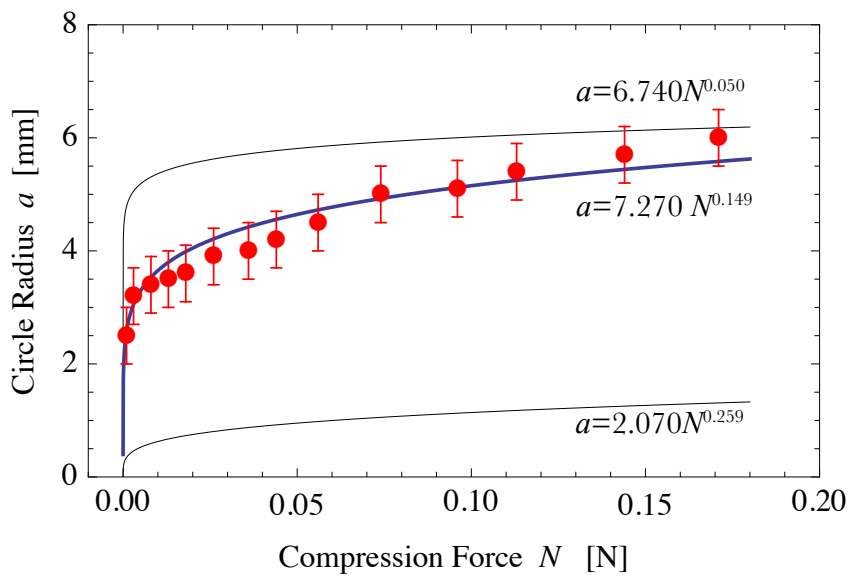

Fig. 9. Compression force $N$ vs contact circle radius $a$, experimental data and nonlinear function $a=g(N)$. The curve $a=7.270$ $N^{0.149}$ obtained for the multi-DoF HC-DEA is in the range set by the curves [17] $a=2.070 N^{0.259}$ relating to a silicone finger and $a=6.740 N^{0.050}$ relating to a viscoelastic finger.

\section{Flat object roto-translation experiments}

In this section we describe the experiments performed to prove the capability of a single multi-DoF HC-DEA to translate a flat object. We also performed an actuation test with an array of two multi-DoF HC-DEAs, thereby roto-translating the flat object. The results from the experiments were merely for validating the actuation concept proposed in the previous sections.

\subsection{Driving electronics}

To drive the multi-DoF HC-DEAs during the experiments, a custom-made electronics consisting of 4 modules powered by a $9 \mathrm{~V}$ battery was developed. Every module consisted of one channels; each channel was composed by a single high voltage power MOSFET (IXTF02N450, Ixys USA), a MOSFET driver (TC1427CPA, Microchip Technology, USA) and a $50 \mathrm{M} \Omega$ resistor (Fig. 10).

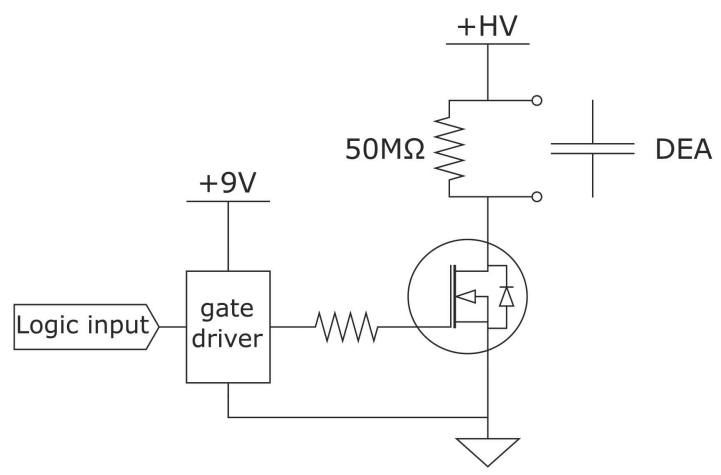

Fig. 10. The modular electronic circuit employed for the activation of each multi-DoF HC-DEA. 


\section{Smart Materials \& Structures}

The MOSFET, in a low side configuration (i.e.: when the load is between the power source and the MOSFET) worked as a switch, allowing for the maximum actuation voltage of $4.5 \mathrm{kV}$ to be provided to the one actuator. A transistor-transistor-logic (TTL) signal (generated according to the actuation pattern) controlled the MOSFET driver, which worked as an interface allowing for a rapid and complete switching of the MOSFET gate. The $50 \mathrm{M} \Omega$ resistor was placed in parallel to each actuator to provide a discharge path, enabling the actuator to quickly return to its resting position when switched off. A four series-connected 8 bit serial-in parallel-out shift register was added to the system so as to enable the device to be controlled using a single serial interface instead of 28 GPIO pins. The signals, used to control the actuation cycles described in Sect. 2, were generated using an Arduino Due.

\subsubsection{Translation test}

In order to verify the ability of a single multi-DoF actuator to translate a flat object, a translation test was performed. A Petri dish with a weight of $8 \mathrm{~g}$ and a diameter of $90 \mathrm{~mm}$ was placed on the actuator's support and used as the object to be displaced during the experiment. The motion of the Petri dish placed on the support structure was triggered by the actuation cycle described in Sect. 2.3, which was set to last 4 seconds (1 second per step). Following the application of the $3.5 \mathrm{kV}$ driving voltage, the actuator induced a horizontal translation of the Petri Dish of $3 \mathrm{~mm}$ for each actuation cycle.

\subsubsection{Roto-translation test}

While in the previous experiment the translation of the Petri dish was obtained with just one actuator by applying the frictional force to the object, in order to achieve the roto-translation motion a torque was also required. As shown in Fig. 11, two multi-DoF HC-DEAs were thus enclosed in a common support structure and secured through adjustment screws at a mutual distance of $70 \mathrm{~mm}$. The support structure consisted of a 3D printed ABS plastic plane with a distribution of holes with a $20 \mathrm{~mm}$ diameter on which the Petri dish (the same Petri dish used for the translation experiment) was placed during the test.

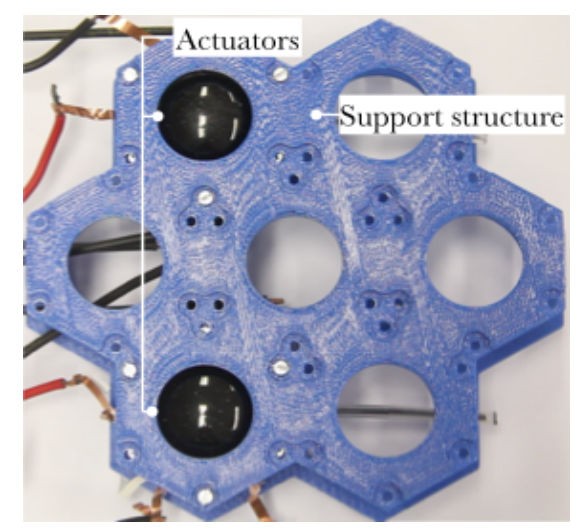

Fig. 11. Setup used for the roto-translation test. Two multi-DoF HC-DEAs enclosed in the support structure used for the experiment. 


\section{Smart Materials \& Structures}

In order to induce the roto-translation of the Petri dish placed on the surface of the support structure, the two actuators of the array represented in Fig.11 were activated with a combination of multiple translation and rotation phases as described in the following. In particular, during each translation phase, both actuators operated clockwise, by repeating the actuation cycle described in Fig. 4, resulting in a translation of the Petri Dish of about $3 \mathrm{~mm}$ along the x-axis. On the other hand, during each rotation phase while one actuator operated following the actuation cycle clockwise, the other actuator reversed its motion operating in the anticlockwise direction. Since the lever distance between the two actuators was $70 \mathrm{~mm}$, this resulted in the generation of a torque on the Petri dish, due to which a rotation of about $2.25^{\circ}$ per cycle was achieved. Figure 12 shows a still frame sequence of the roto-translation test and graphical representation of the actuation control sequence. The test consisted of 5 steps of actuation each lasting 16 seconds, namely (b), (c), (d), (e) and (f) as shown in Fig. 12. During each step both actuators performed Rotation phases (R), or Translation phases (T) according to the sequence reported in Fig. 12. For each phase we also reported whether the actuator was Activated Clockwise (AC) or Activated Anticlockwise (AA).

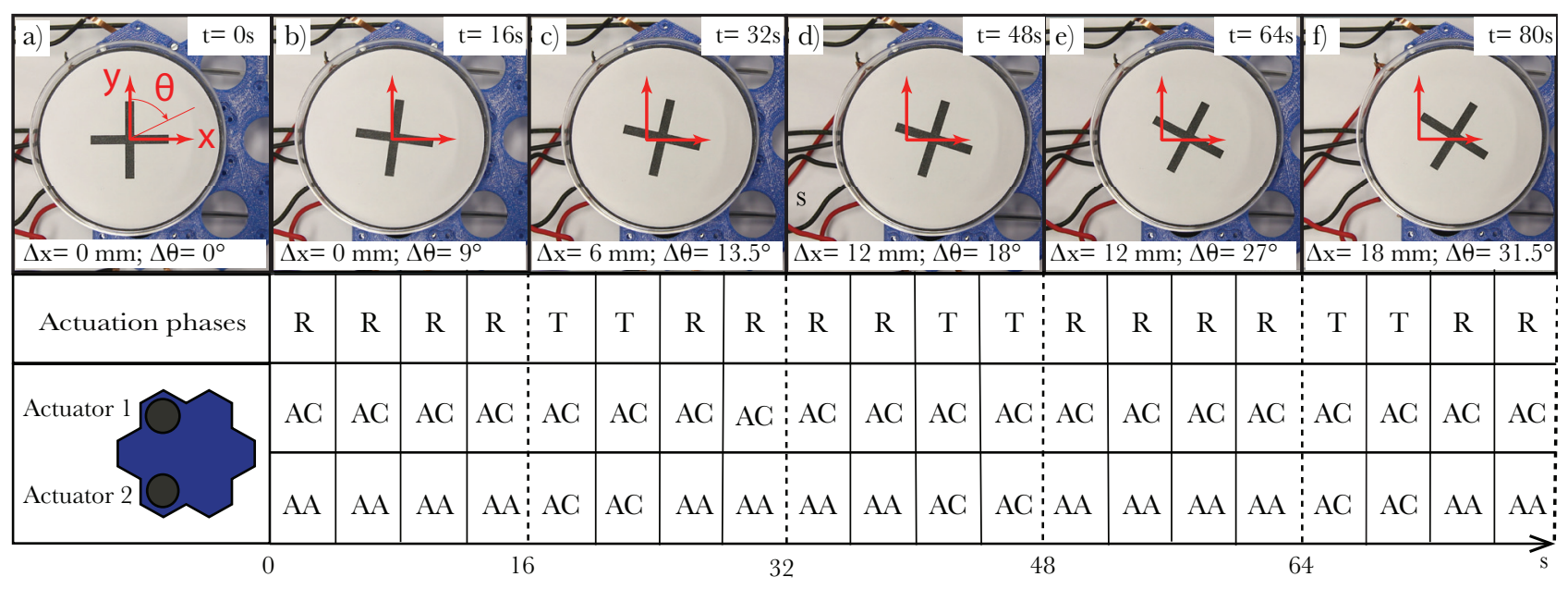

Fig. 12 Roto-translation sequence of a Petri Dish on an array of two multi-DoF HC-DEAs. Starting from the rest position (a), in each of the 5 steps of actuation (b), (c), (d), (e) and (f) both actuators performed rotation phases $\mathbf{R}$, or translation phases $\mathbf{T}$. When both actuators were Activated Clockwise (AC) a translation phase $\mathbf{T}$ was performed, resulting in a translation of the petri dish along the $x$-axis $\Delta x=3 \mathrm{~mm}$. On the other hand, when the Actuator 1 was Activated Anticlockwise (AA), a rotation phase $R$ was performed, resulting in a clockwise rotation of the petri dish $\Delta \theta=2.25^{\circ}$.

\section{Discussion and Conclusions}

In this paper we have presented an upgraded version of the Hydrostatically Coupled Dielectric Elastomer Actuator, which exploits the individual activation of four independent electrodes to achieve a tridimensional motion of its electroactive surface. This feature endows the actuator with a multi-degree of freedom 


\section{Smart Materials \& Structures}

kinematics that allows for the simultaneous generation of both a normal and a tangent action. Indeed, the simultaneous generation of both normal and tangential (frictional) forces distinguishes the proposed actuator from the state of the art, where only push-pull HC-DEAs are proposed.

To demonstrate the capability of the multi-DoF HC-DEA for its use in the roto-translation of flat objects, an experimental characterization was carried out and phenomenological best fitting laws have been proposed. In particular, both the normal and the tangent blocking forces, which are functions of the driving voltage $V$, were derived from such laws. In addition, the relation between the contact area and the normal force acting at the interface with the object was obtained by fitting the experimental data with an analytical function describing an extension of the contact mechanics law of Hertz. This relation showed that the actuator features a gentle touch similar to the soft silicone fingers used in robotics, allowing for its use with delicate objects. Actuation tests were also conducted to assess the effectiveness of the multi-DoF HC-DEA in displacing and rotating a flat object.

In fact, the soft platforms based on the push-pull HC-DEAs reported in literature can only displace round objects, specifically by triggering their rolling. On the other hand, we demonstrated that by using a minimum of two multi-DoF HC-DEA it is possible to also displace flat objects in a controllable manner. Therefore, the proposed actuator is suitable for designing modular soft platforms for roto-translating objects. It can also be adjusted in size and geometry in relation to the shape, dimensions and weight of the object to displace.

Of particular interest for such systems is the dimensional scalability. While HC-DEAs with a diameter in the millimeter scale are reported to be effective [19], to the best of the authors' knowledge there are no reports in the literature regarding an HC-DEA with a diameter over few centimeters. Given that there is likely to be a limit value for the actuator's diameter beyond which the weight of the coupling fluid would hamper its actuation capability; further studies are being carried out.

\section{Acknowledgements}

The work carried out by Aurora De Acutis, Vinicio Tincani, Antonio Bicchi and Danilo De Rossi was supported by the European Commission with Grants No. 645599 "SOMA” and No. 688857 (SoftPro).

The work carried out by Nicola Maria Pugno was supported by the European Commission H2020 under the Graphene Flagship Core 1 No. 696656 (WP14 "Polymer composites") and under the FET Proactive "Neurofibres" No. 732344. The authors gratefully acknowledge the support of Profs. Federico Carpi and Massimiliano Gei as well as Dr. Gabriele Frediani.

\section{References}

[1] Hägele M, Nilsson K, Pires JN, Bischoff R. Industrial Robotics. InSpringer Handbook of Robotics 


\section{Smart Materials \& Structures}

2016 (pp. 1385-1422). Springer International Publishing.

[2] Wang T, Zhang J, Hong J, Wang MY. Dielectric Elastomer Actuators for Soft Wave-Handling Systems. Soft Robotics. 2017 Mar 1;4(1):61-9.

[3] Lee C, Kim M, Kim YJ, Hong N, Ryu S, Kim HJ, Kim S. Soft robot review. International Journal of Control, Automation and Systems. 2017 Feb 1;15(1):3-15.

[4] Hughes J, Culha U, Giardina F, Guenther F, Rosendo A, Iida F. Soft manipulators and grippers: a review. Frontiers in Robotics and AI. 2016; 3:69.

[5] Brown E, Rodenberg N, Amend J, Mozeika A, Steltz E, Zakin M R, Jaeger H M. Universal robotic gripper based on the jamming of granular material. Proceedings of the National Academy of Sciences. 2010; 107.44: 18809-18814.

[6] GmbH FH. WaveHandling [Internet]. Available from: https://www.festo.com/net/SupportPortal/Files/248127/Festo_WaveHandling_en.pdf

[7] Stommel M, Xu W, Lim PP, Kadmiry B. Robotic sorting of ovine offal: Discussion of a soft peristaltic approach. Soft Robotics. 2014 Dec 1;1(4):246-54.

[8] Mosadegh B, Mazzeo A D, Shepherd R F, Morin S A, Gupta U, Sani I Z, Whitesides G M. Control of soft machines using actuators operated by a Braille display. Lab on a Chip, 2014, 14.1: 189-199.

[9] Deng Z, Stommel M, Xu W. A novel soft machine table for manipulation of delicate objects inspired by caterpillar locomotion. IEEE/ASME Transactions on Mechatronics. 2016 Jun;21(3):1702-10.

[10] Majidi C. Soft robotics: a perspective-current trends and prospects for the future. Soft Robotics. 2014 Mar 1;1(1):5-11.

[11] Carpi F, Frediani G, De Rossi D. Hydrostatically coupled dielectric elastomer actuators. IEEE/ASME Transactions On Mechatronics. 2010 Apr;15(2):308-15.

[12] Conn AT, Rossiter J. Towards holonomic electro-elastomer actuators with six degrees of freedom. Smart Materials and Structures. 2012 Feb 10;21(3):035012.

[13] Tröls A, Kogler A, Baumgartner R, Kaltseis R, Keplinger C, Schwödiauer R, Graz I, Bauer S. Stretch dependence of the electrical breakdown strength and dielectric constant of dielectric elastomers. Smart Materials and Structures. 2013 Sep 19;22(10):104012.

[14] Carpi F, Kornbluh R, Sommer-Larsen P, De Rossi D, Alici G. Guest editorial introduction to the focused section on electroactive polymer mechatronics. IEEE/ASME Transactions on Mechatronics. $2011 \mathrm{Feb} ; 16(1): 1-8$. 


\section{Smart Materials \& Structures}

[15] Pelrine R, Kornbluh R, Pei Q, Joseph J. High-speed electrically actuated elastomers with strain greater than 100\%. Science. 2000 Feb 4;287(5454):836-9.

[16] Carpi F, De Rossi D, Kornbluh R, Pelrine RE, Sommer-Larsen P, editors. Dielectric elastomers as electromechanical transducers: fundamentals, materials, devices, models and applications of an emerging electroactive polymer technology. Elsevier; 2011 Sep 6.

[17] Xydas N, Kao I. Modeling of contact mechanics and friction limit surfaces for soft fingers in robotics, with experimental results. The International Journal of Robotics Research. 1999 Sep;18(9):941-50.

[18] Dzidek BM, Adams MJ, Andrews JW, Zhang Z, Johnson SA. Contact mechanics of the human finger pad under compressive loads. Journal of The Royal Society Interface. 2017 Feb 1;14(127):20160935.

[19] Carpi F, Frediani G, Tarantino S, De Rossi D. Millimetre-scale bubble-like dielectric elastomer actuators. Polymer International. 2010 Mar 1;59(3):407-14. 